\title{
Temperaturüberwachung beim Lacktrocknen
}

\author{
Speziell für die Lacktrocknung und -härtung wurde ein neues Messsystem entwickelt. \\ Damit lassen sich nicht nur Härtungsprozesse kontrollieren und bewerten, sondern auch \\ zertifizierte Profilreports erstellen.
}

—ür die Temperaturüberwachung in ren bietet der Oven Tracker XL2 von Datapaq eine bedienfreundliche Lösung. Das speziell speziell auf die Lacktrocknung und -härtung in der Automobilindustrie zugeschnittene System wird auch in weiteren Anwendungen, beispielsweise in der Hochtemperaturbeschichtung bis über $300{ }^{\circ} \mathrm{C}$ sowie bei der Prüfung von Öfen, erfolgreich eingesetzt. Mit den Software-Paketen „Insight Professional“ und „Insight Basic“ können alle Nutzer - vom technischen Experten bis zum Anlagenbediener - Härtungsprozesse bewerten und kontrollieren und einen zertifizierten Profilreport (Qualicoat, ISO9000, CQI-12) erstellen.

Der zweiteilige Datenlogger besteht aus einem 6-oder 8-kanaligen Messwertwandler, der über ein Schnittstellenmodul bis zu 16 Thermoelemente vom Typ K kontaktiert, und einem abnehmbaren „MemoryPaq-Modul“.

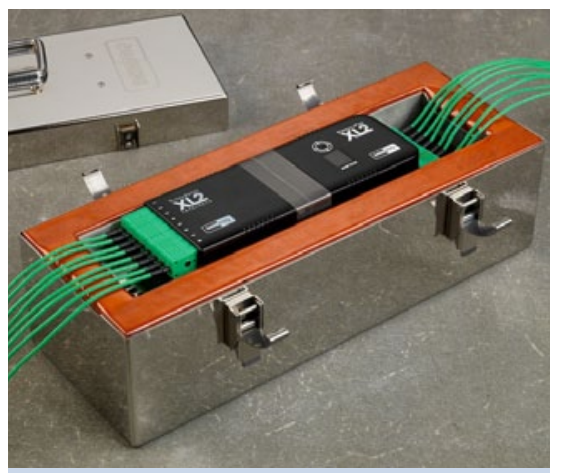

Ein optionales DIB-Schnittstellenmodul („Dual Interface Block“) erleichtert das Kabelmanagement, zum Beispiel beim Messaufbau in einer Lackiererei, in der Autokarosserien lackiert werden
Letzteres zeigt mit einer grünen und einer roten LED direkt am Ofenausgang auf einen Blick an, ob programmierte Prozesskriterien eingehalten wurden.

Diese unmittelbare Qualitätsbewertung erlaubt es Anwendern, etwaige Probleme frühzeitig zu identifizieren. Kontrollkriterien können zum Beispiel der Zeitpunkt bei Erreichen einer Zieltemperatur, die Höchsttemperatur oder der Aushärteindex sein. Der Aushärteindex (oder Datapaq-Wert), von dem Hersteller selbst auf Basis langjähriger Branchenerfahrung entwickelt, ist ein eindeutiger Maßstab für die Oberflächengüte, die von verschiedensten Faktoren wie Schichtdicke, Wärmeleitfähigkeit, Ofenposition und Prozessgeschwindigkeit abhängt.

Für die detaillierte Analyse erstellt das MemoryPaq-Modul außerdem komplette Temperaturprofile. Um diese auszulesen, muss es nicht nach jedem Messlauf an einen Computer angeschlossen werden, da der Speicher für bis zu zehn aufeinanderfolgende Profilaufzeichnungen ausreicht. Dies spart Zeit und Aufwand bei vollständiger Transparenz für die Qualitätssicherung und die Dokumentation.

Bei der Produktentwicklung wurde zudem auf einen bequemen Messaufbau geachtet. So vereinfacht die Möglichkeit, je acht Thermoelemente nach vorn und hinten aus dem Hitzeschutzbehälter zu führen, das Kabelmanagement im Automobilbau erheblich. Auch an schwer zugänglichen Stellen der Karosserie lassen sich die Sensoren komfortabel platzieren.

Für den Oven Tracker XL2 ist eine Auswahl an anwendungsspezifischen

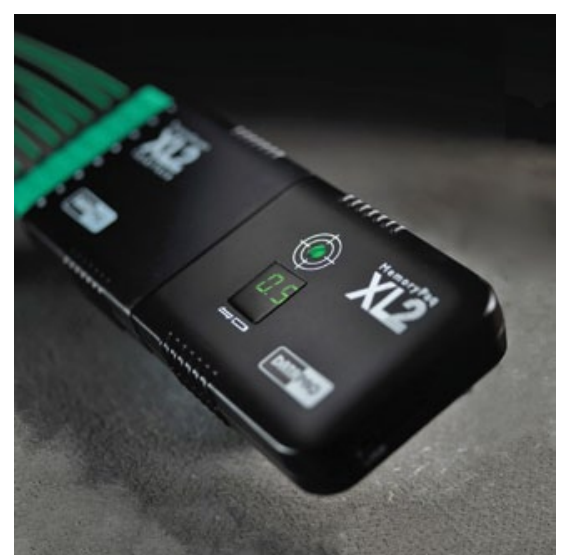

Ein grünes LED-Licht zeigt sofort beim Verlassen des Ofens einen erfolgreichen Durchlauf an

Hitzeschutzbehältern verfügbar. Der XL2-Standardbehälter wurde ebenfalls speziell für Lackierprozesse in der Automobilindustrie entwickelt. Die silikonfreie Konstruktion verhindert Verunreinigungen sowie Lackierfehler und trägt so dazu bei, die geforderte hohe Qualität sicherzustellen. Das XL2-System enthält außerdem eine umfassende Auswahl an Thermoelementen für alle Prozesse und Produkte, zum Beispiel glasfaser- und mineralisolierte Kabeloptionen für Prozesse mit höheren Temperaturen.

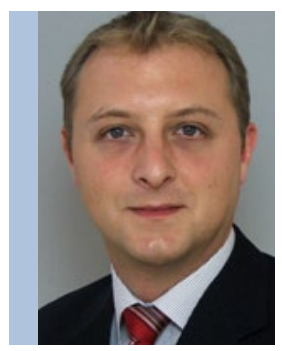

Stefan Oberhuber Regional Sales Manager, Datapaq, Berlin, Tel. 030 478008-0, www.datapaq.com 